\section{Check for updates}

Cite this: Chem. Sci., 2019, 10, 8685

๑ All publication charges for this article have been paid for by the Royal Society of Chemistry

Received 1st June 2019

Accepted 3rd August 2019

DOI: $10.1039 / c 9 s c 02667 c$

rsc.li/chemical-science

\title{
Reversible nanocluster structure transformation between face-centered cubic and icosahedral isomers $\uparrow$
}

\author{
Xi Kang, (D) ${ }^{a}$ Li Huang, ${ }^{b}$ Wei Liu, ${ }^{b}$ Lin Xiong, ${ }^{c}$ Yong Pei, (D) ${ }^{c}$ Zhihu Sun, (D)*b \\ Shuxin Wang, (D) *a Shiqiang Wei ${ }^{\mathrm{b}}{ }^{\mathrm{b}}$ and Manzhou Zhu (D) *a
}

Structural transformations between isomers of nanoclusters provide a platform to tune their properties and understand the fundamental science due to their intimate structure-property correlation. Herein, we demonstrate a reversible transformation between the face-centered cubic (FCC) and icosahedral isomers of $\mathrm{Pt}_{1} \mathrm{Ag}_{28}$ nanoclusters accomplished in the ligand-exchange processes. Ligand-exchange of 1adamantanethiolate protected $\mathrm{Pt}_{1} \mathrm{Ag}_{28}$ by cyclohexanethiolate could transform the $\mathrm{FCC}$ kernel to the icosahedral isomer. Interestingly, the icosahedral $\mathrm{Pt}_{1} \mathrm{Ag}_{28}$ could be reversibly transformed to the $\mathrm{FCC}$ configuration when the cyclohexanethiolate ligand is replaced again by 1 -adamantanethiolate. A combination of UV-vis absorption, mass spectrometry, photo-luminescence and X-ray absorption fine structure unambiguously identifies that the $\mathrm{FCC}$-to-icosahedral structure transformation of $\mathrm{Pt}_{1} \mathrm{Ag}_{28}$ involves two distinct stages: (i) ligand-exchange induced outmost motif transformation and (ii) abrupt innermost kernel transformation. As a result of this structural transformation, the emission wavelength of $\mathrm{Pt}_{1} \mathrm{Ag}_{28}$ red-shifts from 672 to $720 \mathrm{~nm}$, and the HOMO-LUMO energy gap reduces from 1.86 to $1.74 \mathrm{eV}$. This work presents the first example of nanocluster isomers with inter-switching configurations, and will provide new insights into manipulating the properties of nanoclusters through controllably tuning their structures.

\section{Introduction}

Nanoclusters, with the advantages of precise compositions and well-defined structures, provide an exciting opportunity to grasp the structure-property correlation at the atomic level., ${ }^{\mathbf{1} 2}$ The quantum size effect of nanoclusters endows them with a plethora of properties, such as photo-luminescence (PL), catalysis, chirality, and magnetism, to name a few. ${ }^{1,2}$ The property manipulation at the atomic level has long been a hot

${ }^{a}$ Department of Chemistry and Center for Atomic Engineering of Advanced Materials, Anhui Province Key Laboratory of Chemistry for Inorganic/Organic Hybrid Functionalized Materials, Anhui University, Hefei, Anhui, 230601, China. E-mail: ixing@ahu.edu.cn; zmz@ahu.edu.cn

${ }^{b}$ National Synchrotron Radiation Laboratory, University of Science and Technology of China, Hefei, Anhui, 230029, China. E-mail: zhsun@ustc.edu.cn

'Department of Chemistry, Key Laboratory of Environmentally Friendly Chemistry and Applications of Ministry of Education, Xiangtan University, Xiangtan, Hunan, 411105, China

$\dagger$ Electronic supplementary information (ESI) available: Fig. S1-S23 showing the total structure, TGA and XPS results of $\mathbf{P t}_{1} \mathbf{A g}_{28} \mathbf{2}^{-2}$; the stability test; DPV results; catalytic activity results; TEM images; structural comparison between $\mathbf{P t}_{\mathbf{1}} \mathbf{A g}_{\mathbf{2 8}} \mathbf{- 1}$ and $\mathbf{P t}_{1} \mathbf{A g}_{28}$-2; time-dependent UV-vis spectra, PL spectra and ESI-MS results from $\mathbf{P t}_{\mathbf{1}} \mathbf{A g}_{28} \mathbf{2}$ to $\mathbf{P t}_{\mathbf{1}} \mathbf{A g}_{28} \mathbf{- 1}$; detailed XAFS results; TDDFT results; temperature-dependent PL and UV-vis spectra of $\mathbf{P t}_{1} \mathbf{A g}_{28} \mathbf{2}^{-2}$; the crystal data and structure refinement for $\mathbf{P t}_{1} \mathbf{A g}_{28}$-2. CCDC 1840953. For ESI and crystallographic data in CIF or other electronic format see DOI: $10.1039 / \mathrm{c} 9 \mathrm{sc} 02667 \mathrm{c}$ topic, and has allowed a series of nanoclusters with controllable

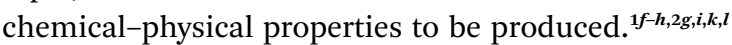

Isomerism is being intensely pursued in nanoscience, and has been exploited for tailoring the performances of nanoparticles. ${ }^{3}$ However, understanding the isomerism phenomenon at the atomic level has been largely impeded due to the poly-disperse sizes as well as the uncertain surface coordination modes of nanoparticles. ${ }^{\mathbf{1 a}, \boldsymbol{b}, \boldsymbol{3} \boldsymbol{b}}$ The precise structures of nanoclusters make it possible to fully grasp the isomerism phenomenon. ${ }^{\mathbf{4}-\mathbf{6}}$ Although stereoisomerism has been extensively studied in the nanocluster field (that is, obtaining chiraloptical nanoclusters via the separation of left- and right-handed isomers), ${ }^{4,5}$ structural isomerism remains rare. Up to now, only a few structural isomers in the nanocluster range have been observed (e.g., $\mathrm{Au}_{28}(\mathrm{SR})_{20}, \mathrm{Au}_{38}(\mathrm{SR})_{24}$, and $\left.\mathrm{Au}_{52}(\mathrm{SR})_{32}\right) \cdot{ }^{6 a, c-f}$ Different structure-dependent properties (e.g., optical absorption, catalytic activity, and thermal stability) have been investigated on the basis of these nanocluster isomers. ${ }^{5 f, 6}$ In addition, experimental and theoretical efforts in exploring the isomerism effect on atomic arrangement and properties of nanoclusters should be continued because such findings enable us to fully grasp the structure-property correlation, and thus help us to design new nanoclusters with unique functions. ${ }^{5,6}$

Meanwhile, accompanied by the structure determination of nanoclusters resolved by single-crystal X-ray crystallography 
(SCXC), several efforts have been made for grasping the sizegrowth and structure-transformation modes from small complexes to nanoclusters, then to large nanoparticles. ${ }^{1, n, 7}$ Icosahedron and face-centered cubic (FCC) are the two most common configurations of nanocluster kernels. The structural transformation from an icosahedral to FCC configuration or its reverse process has been reported recently in the nanocluster field. ${ }^{6 d, 8}$ For instance, ligand-exchanging $\mathrm{Au}_{25}\left(\mathrm{~S}_{-}-\mathrm{C}_{2} \mathrm{H}_{4} \mathrm{Ph}\right)_{18}$ with excess ${ }^{t} \mathrm{BuPh}-\mathrm{SH}$ changes the icosahedral configuration of the $\mathrm{Au}_{13}$ kernel into a FCC configuration. ${ }^{6 \boldsymbol{d}}$ Besides, the existence of $\mathrm{Me}_{2} \mathrm{PhSH}$ can transform $\mathrm{Au}_{44}\left(\mathrm{~S}-\mathrm{Ph}^{t} \mathrm{Bu}\right)_{28}$ into $\mathrm{Au}_{44}\left(\mathrm{~S}-\mathrm{PhMe}_{2}\right)_{26}$, where the kernel changes from the FCC to the icosahedral configuration. ${ }^{8 a}$ However, for nanocluster isomer systems, to the best of our knowledge, the structural transformation from the FCC to icosahedron or its reverse process, not to mention the reversible transformation between these two configurations, remains incomplete. Such a shortage impedes the full understanding of nanocluster isomerism in terms of structuretransformation modes and structure-property correlation.

In the current work, a reversible transformation has been accomplished between FCC and icosahedral isomers of $\mathrm{Pt}_{1} \mathrm{Ag}_{28}$ nanoclusters. The ligand-exchange method is herein exploited for fulfilling the reversible transformation between $\mathrm{Pt}_{1} \mathrm{Ag}_{28}(\mathrm{~S}$ $\mathrm{Adm})_{18}\left(\mathrm{PPh}_{3}\right)_{4} \quad\left(\mathbf{P t}_{\mathbf{1}} \mathbf{A g}_{28} \mathbf{- 1}\right.$, where S-Adm represents 1-adamantanethiol) with a FCC $\mathrm{Pt}_{1} \mathrm{Ag}_{12}$ kernel and $\mathrm{Pt}_{1} \mathrm{Ag}_{28}(\mathrm{~S}-\mathrm{c}$ $\left.\mathrm{C}_{6} \mathrm{H}_{11}\right)_{18}\left(\mathrm{PPh}_{3}\right)_{4}\left(\mathbf{P t}_{\mathbf{1}} \mathbf{A g}_{28}-\mathbf{2}\right.$, where $\mathrm{S}-c-\mathrm{C}_{6} \mathrm{H}_{11}$ represents cyclohexanethiol) with an icosahedral $\mathrm{Pt}_{1} \mathrm{Ag}_{12}$ kernel. ESI-MS, PL and EXAFS results are combined to demonstrate that the configurational transformation between the FCC and the icosahedron contains two distinct stages: the motif transformation process and kernel transformation process, where the latter transformation is induced by the former one. Accompanied by the structural transformation (from $\mathbf{P t}_{\mathbf{1}} \mathbf{A g}_{28} \mathbf{- 1}$ to $\mathbf{P t}_{\mathbf{1}} \mathbf{A g}_{\mathbf{2 8}} \mathbf{- 2}$ ), the emission wavelength red-shifts from $672 \mathrm{~nm}$ to $720 \mathrm{~nm}$, and the HOMO-LUMO energy gap reduces from $1.86 \mathrm{eV}$ to $1.74 \mathrm{eV}$.

\section{Experimental methods}

\subsection{Materials}

All chemicals including silver nitrate $\left(\mathrm{AgNO}_{3}, 99.9 \%\right.$, metal basis), hexachloroplatinic(Iv) acid $\left(\mathrm{H}_{2} \mathrm{PtCl}_{6} \cdot 6 \mathrm{H}_{2} \mathrm{O}, 99.9 \%\right.$ metal basis), triphenylphosphine $\left(\mathrm{PPh}_{3}, 99 \%\right)$, 1-adamantanethiol (Adm-SH, $\mathrm{C}_{10} \mathrm{H}_{15} \mathrm{SH}, 99 \%$ ), cyclohexanethiol $\left(\mathrm{C}_{6} \mathrm{H}_{11}-c-\mathrm{SH}, 99 \%\right.$ ), sodium borohydride $\left(\mathrm{NaBH}_{4}, 99.9 \%\right)$, styrene $\left(\mathrm{C}_{6} \mathrm{H}_{5}-\mathrm{C}_{2} \mathrm{H}_{3}\right.$, 99.5\%), potassium carbonate $\left(\mathrm{K}_{2} \mathrm{CO}_{3}, 99.5 \%\right)$, methylene chloride $\left(\mathrm{CH}_{2} \mathrm{Cl}_{2}\right.$, HPLC grade), methanol $\left(\mathrm{CH}_{3} \mathrm{OH}, \mathrm{HPLC}\right)$, acetic ether $\left(\mathrm{CH}_{3} \mathrm{COOC}_{2} \mathrm{H}_{5}\right.$, HPLC), ether $\left(\left(\mathrm{C}_{2} \mathrm{H}_{5}\right)_{2} \mathrm{O}\right.$, HPLC), and toluene $\left(\mathrm{C}_{6} \mathrm{H}_{5}-\mathrm{CH}_{3}\right.$, HPLC) were purchased from Sigma-Aldrich and used without further purification. All glassware were thoroughly cleaned with aqua regia $\left(\mathrm{HCl}: \mathrm{HNO}_{3}=3: 1 \mathrm{v} / \mathrm{v}\right)$, rinsed with copious pure water, and then dried in an oven prior to use.

\subsection{Synthesis of $\mathrm{Pt}_{1} \mathrm{Ag}_{28}-1$ nanoclusters}

The synthesis of $\mathbf{P t}_{\mathbf{1}} \mathbf{A g}_{\mathbf{2 8}} \mathbf{- 1}$ nanoclusters was carried out with reference to our previous work. ${ }^{8 b}$

\subsection{Converting $\mathrm{Pt}_{1} \mathrm{Ag}_{28}$-1 into $\mathrm{Pt}_{1} \mathrm{Ag}_{28}{ }^{-2}$ nanoclusters}

Specifically, $10 \mathrm{mg}$ of $\mathbf{P t}_{\mathbf{1}} \mathbf{A g}_{\mathbf{2 8}} \mathbf{- 1}$ nanoclusters was dissolved in $30 \mathrm{~mL}$ of $\mathrm{CH}_{2} \mathrm{Cl}_{2} .300 \mu \mathrm{L}$ of $\mathrm{C}_{6} \mathrm{H}_{11}{ }^{-c}-\mathrm{SH}$ was added and reacted for 2 hours at $40{ }^{\circ} \mathrm{C}$. After the reaction was complete, the solution was rotavaporated, and then approximately $50 \mathrm{~mL}$ of methanol was added to wash the product. The precipitate was dissolved in $\mathrm{CH}_{2} \mathrm{Cl}_{2}$ giving rise to the solution of $\mathbf{P t}_{\mathbf{1}} \mathbf{A g}_{\mathbf{2 8}} \mathbf{- 2}$ nanoclusters. The yield was about $80 \%$ (i.e., $8 \mathrm{mg}$ of $\mathbf{P t}_{\mathbf{1}} \mathbf{A g}_{\mathbf{2 8}} \mathbf{- 2}$ was obtained).

\subsection{Converting $\mathrm{Pt}_{1} \mathrm{Ag}_{28}$-2 into $\mathrm{Pt}_{1} \mathrm{Ag}_{28}$-1 nanoclusters}

Specifically, $10 \mathrm{mg}$ of $\mathbf{P t}_{\mathbf{1}} \mathbf{A g}_{\mathbf{2 8}} \mathbf{- 2}$ nanoclusters was dissolved in $30 \mathrm{~mL}$ of $\mathrm{CH}_{2} \mathrm{Cl}_{2} .0 .1 \mathrm{~g}$ of Adm-SH was added and reacted for 3 minutes at room temperature. After the reaction was complete, the solution was rotavaporated, and then approximately $50 \mathrm{~mL}$ of methanol was added to wash the product. The precipitate was dissolved in $\mathrm{CH}_{2} \mathrm{Cl}_{2}$ giving rise to the solution of $\mathbf{P t}_{\mathbf{1}} \mathbf{A g}_{\mathbf{2 8}} \mathbf{- 1}$ nanoclusters. The yield was about $90 \%$ (i.e., $9 \mathrm{mg}$ of $\mathbf{P t}_{\mathbf{1}} \mathbf{A g}_{\mathbf{2 8}} \mathbf{- 1}$ was obtained). Compared with the conversion from $\mathbf{P t}_{\mathbf{1}} \mathbf{A g}_{\mathbf{2 8}} \mathbf{- 1}$ to $\mathbf{P t}_{\mathbf{1}} \mathbf{A g}_{\mathbf{2 8}} \mathbf{- 2}$, the reverse process is quicker.

\subsection{Crystallization of the $\mathrm{Pt}_{1} \mathrm{Ag}_{28}$-2 nanoclusters}

Single crystals of $\mathbf{P t}_{\mathbf{1}} \mathbf{A g}_{\mathbf{2 8}}$-2 were crystallized by vapor diffusion of ether into the $\mathrm{CH}_{2} \mathrm{Cl}_{2}$ solution of the nanoclusters over 7 days. For promoting the crystallization, $\mathrm{Na}^{+}\left(\mathrm{BPh}_{4}\right)^{-}$counterions (molar ratio between clusters and counter-ions was $1: 2$ ) were added into the $\mathrm{CH}_{2} \mathrm{Cl}_{2}$ solution. Then black crystals were collected and the structure of the $\mathbf{P t}_{\mathbf{1}} \mathbf{A g}_{28}$-2 nanocluster was determined. The CCDC number of the $\mathbf{P t}_{\mathbf{1}} \mathbf{A g}_{\mathbf{2 8}} \mathbf{- 2}$ nanocluster is 1840953.

\subsection{Test of the temperature-PL correlation}

$10 \mathrm{mg}$ of $\mathbf{P t}_{\mathbf{1}} \mathbf{A g}_{\mathbf{2 8}} \mathbf{- 1}$ (or $\mathbf{P t}_{\mathbf{1}} \mathbf{A g}_{\mathbf{2 8}}$-2) was dissolved in $10 \mathrm{~mL}$ of $\mathrm{CH}_{2} \mathrm{Cl}_{2} / 2-\mathrm{CH}_{3}-\mathrm{THF}$. Then the solutions were cooled to different temperatures and the PL spectra were measured.

\subsection{Catalytic performance}

For the preparation of catalysts, $\mathbf{P t}_{\mathbf{1}} \mathbf{A g}_{\mathbf{2 8}} \mathbf{- 1}$ ( or $\mathbf{P t}_{\mathbf{1}} \mathbf{A g}_{28}$-2) clusters were supported on commercial carbon nanotubes (CNTs; Beijing Bo Yu high-tech new material technology Co., Ltd). The CNTs were first dispersed in toluene, and the nanoclusters were added to the suspension of CNTs under vigorous magnetic stirring. The adsorption of clusters was allowed to proceed overnight. Then the product was separated from the solution by centrifugation. The cluster@CNT composite was dried in a vacuum for $12 \mathrm{~h}$; then, $\mathbf{P t}_{\mathbf{1}} \mathbf{A g}_{28} \mathbf{- 1} @ \mathrm{CNT}$ and $\mathbf{P t} \mathbf{A}_{\mathbf{1}} \mathbf{A g}_{\mathbf{2 8}} \mathbf{- 2} @ \mathrm{CNT}$ catalysts (with a $2 \mathrm{wt} \%$ cluster loading) were obtained.

For the catalytic activity test, a $10 \mathrm{~mL}$ Schlenk bottle was charged with $0.5 \mathrm{mmol}$ of styrene, $1.5 \mathrm{mmol}$ of TBHP (tert-butyl hydroperoxide), $20 \mathrm{mg}$ of cluster@CNT catalyst, $10 \mathrm{mg}$ of $\mathrm{K}_{2} \mathrm{CO}_{3}$, and $2 \mathrm{~mL}$ of toluene. Then the suspension was stirred at $50{ }^{\circ} \mathrm{C}$ for 24 hours. The suspension was then centrifuged to remove solids, and the catalytic product was analysed by gas chromatography with an internal standard. 


\subsection{XAFS (X-ray absorption fine structure spectroscopy) measurements}

XAFS measurements at the $\mathrm{Pt} \mathrm{L}_{3}$-edge $(11564 \mathrm{eV})$ were performed at the beamline BL14W1 station of the Shanghai Synchrotron Radiation Facility (SSRF), China. The storage ring of the SSRF was working at an energy of $3.5 \mathrm{GeV}$ with an average electron current of $300 \mathrm{~mA}$. The hard X-ray was monochromatized with a Si (311) monochromator. EXAFS data were collected in the transmission mode in the energy range from -200 below to $1000 \mathrm{eV}$ above the $\mathrm{Pt}_{3}$-edge. The acquired EXAFS data were processed according to the standard procedures using the ARTEMIS module implemented in the IFEFFIT software packages.

\subsection{X-ray crystallography}

The data collection for single crystal X-ray diffraction was carried out on a Bruker Smart APEX II CCD diffractometer under liquid nitrogen flow at $200 \mathrm{~K}$, using graphitemonochromatized Mo $\mathrm{K} \alpha$ radiation $(\lambda=0.71073 \AA)$. Data reductions and absorption corrections were performed using the SAINT and SADABS programs, respectively. The electron density was squeezed by Olex 2 . The structure was solved by direct methods and refined with full-matrix least squares on $F^{2}$ using the SHELXTL software package. All non-hydrogen atoms were refined anisotropically, and all the hydrogen atoms were set in geometrically calculated positions and refined isotropically using a riding model.

\section{Theoretical methods}

Density functional theory (DFT) calculations were employed to optimize the geometric structures and calculate the Kohn-Sham orbitals of $\mathrm{Pt}_{1} \mathrm{Ag}_{28}$ nanoclusters using the Perdew-Burke-Ernzerhof (PBE) GGA functional. ${ }^{9}$ The triple-zeta polarized (TZP) basis set with inclusion of the scalar relativistic effect via a zerothorder regular approximation (ZORA) implemented in the ADF package was adopted. ${ }^{10}$ In the electronic structure analysis, the Kohn-Sham orbitals were calculated to analyze contributions of different atomic orbital types to molecular orbitals.

\subsection{Characterization}

UV-vis absorption spectra of nanoclusters dissolved in $\mathrm{CH}_{2} \mathrm{Cl}_{2}$ were recorded using an Agilent 8453 diode array spectrometer.

Electrospray ionization time-of-flight mass spectrometry (ESI-TOF-MS) measurement was performed using a MicrOTOFQIII high-resolution mass spectrometer.

PL spectra were measured on an FL-4500 spectrofluorometer with the same optical density (OD) of $\sim 0.05$. Of note, the PL excitation spectrum of $\mathbf{P t}_{\mathbf{1}} \mathbf{A g}_{28} \mathbf{- 1}$ or $\mathbf{P t}_{\mathbf{1}} \mathbf{A g}_{28} \mathbf{- 2}$ was measured at 600 or $750 \mathrm{~nm}$, respectively, for suppressing the interference from each other (e.g., $\mathbf{P t}_{\mathbf{1}} \mathbf{A g}_{\mathbf{2 8}} \mathbf{- 1}$ fluoresces at $600 \mathrm{~nm}$, while $\mathbf{P t}_{\mathbf{1}} \mathbf{A g}_{28}$-2 does not fluoresce at this wavelength).

Quantum yields (QYs) were measured with dilute solutions of nanoclusters on a HORIBA FluoroMax-4P.

Transmission electron microscopy (TEM) was conducted on a JEM-2100 microscope with an accelerating voltage of $200 \mathrm{kV}$.
Thermogravimetric analysis (TGA) was carried out on a thermogravimetric analyser (DTG-60H, Shimadzu Instruments, Inc.)

X-ray photoelectron spectroscopy (XPS) measurement was performed on a Thermo ESCALAB 250, configured with a monochromated Al $\mathrm{K} \alpha(1486.8 \mathrm{eV}) 150 \mathrm{~W}$ X-ray source, $0.5 \mathrm{~mm}$ circular spot size, flood gun to counter charging effects, and analysis chamber base pressure lower than $1 \times 10^{-9} \mathrm{mbar}$.

Inductively coupled plasma-atomic emission spectrometry (ICP-AES) measurements were performed on an Atomscan Advantage instrument made by Thermo Jarrell Ash Corporation.

Electrochemical measurements (differential pulse voltammetry, DPV) of clusters were performed with an electrochemical workstation (CHI 700E) using a Pt working electrode $0.4 \mathrm{~mm}$ diameter), a Pt wire counter electrode and an $\mathrm{Ag}$ wire quasireference electrode in $0.1 \mathrm{M} \mathrm{Bu}_{4} \mathrm{NPF}_{6}-\mathrm{CH}_{2} \mathrm{Cl}_{2}$. The electrolyte solution was deaerated with ultra-high purity nitrogen for $40 \mathrm{~min}$ and blanketed under a nitrogen atmosphere during the entire experimental procedure.

\section{Results and discussion}

\subsection{Syntheses and crystallization}

The $\mathbf{P t}_{\mathbf{1}} \mathbf{A g}_{28} \mathbf{- 1}$ nanoclusters were prepared using our previously reported procedure. ${ }^{8 b}$ The $\mathbf{P t}_{\mathbf{1}} \mathbf{A g}_{28}$-2 nanoclusters were synthesized by reacting pure $\mathbf{P t}_{\mathbf{1}} \mathbf{A g}_{\mathbf{2 8}} \mathbf{- 1}$ with excess $\mathrm{HS}-c-\mathrm{C}_{6} \mathrm{H}_{11}$ at $40{ }^{\circ} \mathrm{C}$ (see Experimental Methods for more details). After $\sim 2$ hours, all the $\mathbf{P t}_{\mathbf{1}} \mathbf{A g}_{\mathbf{2 8}} \mathbf{- 1}$ nanoclusters were completely converted into $\mathbf{P t}_{\mathbf{1}} \mathbf{A g}_{28} \mathbf{- 2}$ in a high yield ( $>80 \%, \mathrm{Ag}$ atom basis). The as-prepared $\mathbf{P t}_{\mathbf{1}} \mathbf{A g}_{28} \mathbf{- 2}$ was crystallized by vapor diffusion of ether into a $\mathrm{CH}_{2} \mathrm{Cl}_{2}$ solution of the nanoclusters over 7 days. The $\mathbf{P t}_{\mathbf{1}} \mathbf{A g}_{\mathbf{2 8}} \mathbf{- 1}$ structure was reported by us in early work. ${ }^{8 b}$ The structure of $\mathbf{P t}_{1} \mathbf{A g}_{28}-\mathbf{2}$ is newly determined in the current work. X-ray photoelectron spectroscopy (XPS) and inductively coupled plasma (ICP) measurements were performed to validate the ratio of Pt/Ag in the bi-metallic $\mathbf{P t}_{\mathbf{1}} \mathbf{A g}_{\mathbf{2 8}} \mathbf{- 2}$ nanoclusters (Fig. S1, $\mathrm{S} 2$ and Table $\mathrm{S} 1 \dagger)$, and the results perfectly matched the theoretical value (1/28 of $\mathrm{Pt} / \mathrm{Ag}$ ). Furthermore, the purity of $\mathbf{P t}_{\mathbf{1}} \mathbf{A g}_{\mathbf{2 8}} \mathbf{- 2}$ was confirmed by thermogravimetric analysis (TGA). The experimental weight loss of $49.57 \%$ (Fig. S3†) is consistent with the calculated loss (49.26\%) of the ligands (i.e., $\mathrm{PPh}_{3}$ and S-c$\left.\mathrm{C}_{6} \mathrm{H}_{11}\right)$ in $\mathbf{P t}_{\mathbf{1}} \mathbf{A g}_{28}$-2. Both $\mathrm{Pt}_{1} \mathrm{Ag}_{28}$ nanoclusters are highly stable at $50{ }^{\circ} \mathrm{C}$ (Fig. $\mathrm{S} 4 ; \dagger$ clusters are dissolved in $\mathrm{CHCl}_{3}$, and in contact with air). In the DPV analysis (Fig. S5 $\dagger$ ), $\mathbf{P t}_{\mathbf{1}} \mathbf{A g}_{\mathbf{2 8}} \mathbf{- 1}$ showed two oxidation peaks at $0.76 \mathrm{~V}(\mathrm{O} 1)$ and $1.28 \mathrm{~V}(\mathrm{O} 2)$ and a reduction peak at $-1.32 \mathrm{~V}$ (R1); in comparison, $\mathbf{P t}_{\mathbf{1}} \mathbf{A g}_{\mathbf{2 8}} \mathbf{- 2}$ exhibited three oxidation peaks at $0.75 \mathrm{~V}(\mathrm{O} 1), 1.08 \mathrm{~V}(\mathrm{O} 2)$ and $1.45 \mathrm{~V}(\mathrm{O} 3)$ and a reduction peak at $-0.92 \mathrm{~V}(\mathrm{R} 1)$. The catalytic performance of both $\mathrm{Pt}_{1} \mathrm{Ag}_{28}$ nanoclusters in the oxidation of styrene was evaluated (Fig. S6, $\dagger$ and see the catalysis conditions in Experimental methods). The catalysis was performed at $50{ }^{\circ} \mathrm{C}$ to ensure that both clusters were stable. Although the catalytic selectivity for epoxide or benzaldehyde is similar in both catalysis, the overall catalytic conversion by $\mathbf{P t}_{\mathbf{1}} \mathbf{A g}_{\mathbf{2 8}}$-2@CNTs is much higher than that by $\mathbf{P t}_{\mathbf{1}} \mathbf{A g}_{\mathbf{2 8}} \mathbf{- 1}$ @CNTs (66.48\% versus $43.43 \%$; shown in 
Fig. S6†). Such a difference may result from the ligand effect between the two nanoclusters.

The $\mathbf{P t}_{\mathbf{1}} \mathbf{A g}_{28}$-2 nanoclusters crystallize in the $P 2_{1} / c$ space group. The structure of $\mathbf{P t}_{\mathbf{1}} \mathbf{A g}_{\mathbf{2 8}} \mathbf{- 2}$ is shown in Fig. 1A and the structural anatomy is shown in Fig. 1B-E (see Fig. S7† for the complete structure). Specifically, a single Pt atom is arranged in the innermost position of the overall structure (Fig. 1B), which is further capped by an $\mathrm{Ag}_{12}$ cage, forming a $\mathrm{Pt}_{1} \mathrm{Ag}_{12}$ kernel with an icosahedral configuration (Fig. 1C). Furthermore, this $\mathrm{Pt}_{1} \mathrm{Ag}_{12}$ kernel is surrounded by four $\mathrm{Ag}_{3}\left(\mathrm{~S}-c-\mathrm{C}_{6} \mathrm{H}_{11}\right)_{6}$ motifs. Of note, each $\mathrm{Ag}_{3}\left(\mathrm{~S}-c-\mathrm{C}_{6} \mathrm{H}_{11}\right)_{6}$ motif links with three other motifs via sharing the terminal thiolates, which constitutes a cage-like $\mathrm{Ag}_{12}\left(\mathrm{~S}-c-\mathrm{C}_{6} \mathrm{H}_{11}\right)_{18}$ motif structure fully surrounding the $\mathrm{Pt}_{1} \mathrm{Ag}_{12}$ kernel (Fig. 1D). Moreover, four $\mathrm{Ag}-\mathrm{PPh}_{3}$ architectures occupy the four vacancies of the aforementioned $\mathrm{Pt}_{1} \mathrm{Ag}_{12} @ \mathrm{Ag}_{12}(\mathrm{~S}-\mathrm{c}$ $\left.\mathrm{C}_{6} \mathrm{H}_{11}\right)_{18}$ structure, giving rise to the final $\mathbf{P t}_{\mathbf{1}} \mathbf{A g}_{\mathbf{2 8}} \mathbf{- 2}$ structure. It should be noted that the capped $\mathrm{Ag}_{12}(\mathrm{SR})_{18} @\left(\mathrm{Ag}-\mathrm{PPh}_{3}\right)_{4}$

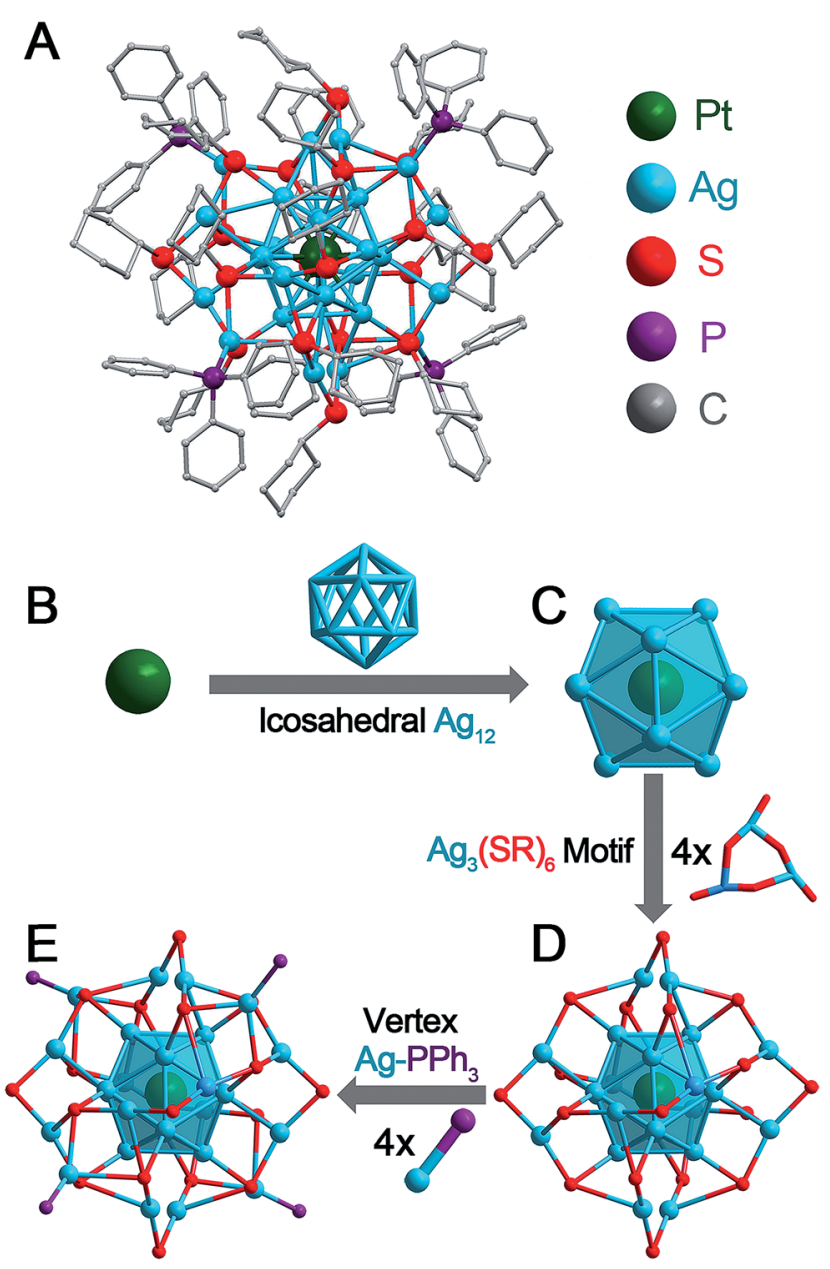

Fig. $1 \mathrm{Pt}_{1} \mathrm{Ag}_{28}-2$ nanocluster and its structural anatomy. (A) Total structure. (B) Innermost Pt atom. (C) $\mathrm{Pt}_{1} \mathrm{Ag}_{12}$ kernel with an icosahedral configuration. (D) $\mathrm{Pt}_{1} \mathrm{Ag}_{12}$ kernel protected by four $\mathrm{Ag}_{3}(\mathrm{~S}-\mathrm{Adm})_{6}$ motifs. These four motifs make up a large $\mathrm{Ag}_{12}(\mathrm{~S}-\mathrm{Adm})_{18}$ motif wrapping the icosahedral kernel. (E) Four $\mathrm{Ag}-\mathrm{PPh}_{3}$ architectures occupy the vertex positions of the nanocluster. Color codes: green sphere, Pt; blue spheres, Ag; red spheres, S; purple spheres, P; and grey spheres, C. For clarity, the hydrogen atoms are not shown. structure of $\mathbf{P t}_{1} \mathbf{A g}_{28}-\mathbf{2}$ is similar to that of $\mathbf{P t}_{1} \mathbf{A g}_{28} \mathbf{- 1}^{\mathbf{8} b}$ The TEM images of $\mathbf{P t}_{\mathbf{1}} \mathbf{A g}_{\mathbf{2 8}} \mathbf{- 1}$ and $\mathbf{P t}_{\mathbf{1}} \mathbf{A g}_{\mathbf{2 8}} \mathbf{- 2}$ indicate that the clusters are uniform in size of about $1.6 \mathrm{~nm}$ (Fig. S8†), and this agrees with that determined by X-ray structural analysis.

\subsection{Reversible structure transformation}

In order to elucidate the structural differences induced by the ligand exchange (i.e., $\mathrm{S}-c-\mathrm{C}_{6} \mathrm{H}_{11}$ versus $\mathrm{S}-\mathrm{Adm}$ ), a comparison of the kernels of $\mathbf{P t}_{\mathbf{1}} \mathbf{A g}_{\mathbf{2 8}} \mathbf{- 1}$ and $\mathbf{P t}_{\mathbf{1}} \mathbf{A g}_{\mathbf{2 8}} \mathbf{- 2}$ is provided in Fig. 2. Both $\mathrm{Pt}_{1} \mathrm{Ag}_{28}$ nanoclusters comprise a $\mathrm{Pt}_{1} \mathrm{Ag}_{12}$ kernel; however, the FCC configuration of the $\mathrm{Pt}_{1} \mathrm{Ag}_{12}$ kernel in $\mathbf{P t}_{\mathbf{1}} \mathbf{A g}_{\mathbf{2 8}} \mathbf{- 1}$ turns into the icosahedral configuration when the nanocluster converts to $\mathbf{P t}_{1} \mathbf{A g}_{28}$-2. The opposite process is also confirmed by reacting $\mathbf{P t}_{\mathbf{1}} \mathbf{A g}_{28}$-2 with excess HS-Adm. Previously, two nanoclusters following the isomerism phenomenon are almost arranged in the same molecular configuration, e.g., $\mathrm{Au}_{21}(\mathrm{~S}-\mathrm{Adm})_{15}$ and $\mathrm{Au}_{21}\left(\mathrm{~S}-{ }^{t} \mathrm{Bu}\right)_{15}$ isomers with a FCC configuration, ${ }^{11,12} \mathrm{Au}_{28}(\mathrm{~S}-$ $\left.\mathrm{Ph}^{t} \mathrm{Bu}\right)_{20}$ and $\mathrm{Au}_{28}\left(\mathrm{~S}-c-\mathrm{C}_{6} \mathrm{H}_{11}\right)_{20}$ isomers with a FCC configuration, ${ }^{6 d, e} \mathrm{Au}_{52}\left(\mathrm{~S}-\mathrm{Ph}^{t} \mathrm{Bu}\right)_{32}$ and $\mathrm{Au}_{52}\left(\mathrm{~S}-\mathrm{PhC}_{2} \mathrm{H}_{4}\right)_{32}$ isomers with a FCC configuration, ${ }^{6 c} \mathrm{Au}_{9} \mathrm{Ag}_{12}(\mathrm{~S}-\mathrm{Adm})_{4}(\mathrm{dppm})_{6} \mathrm{Cl}_{6}$ and $\mathrm{Au}_{9^{-}}$ $\mathrm{Ag}_{12}\left(\mathrm{~S}-{ }^{t} \mathrm{Bu}\right)_{4}(\mathrm{dppm})_{6} \mathrm{Cl}_{6}$ with an icosahedral configuration, ${ }^{6 f}$ and so on.
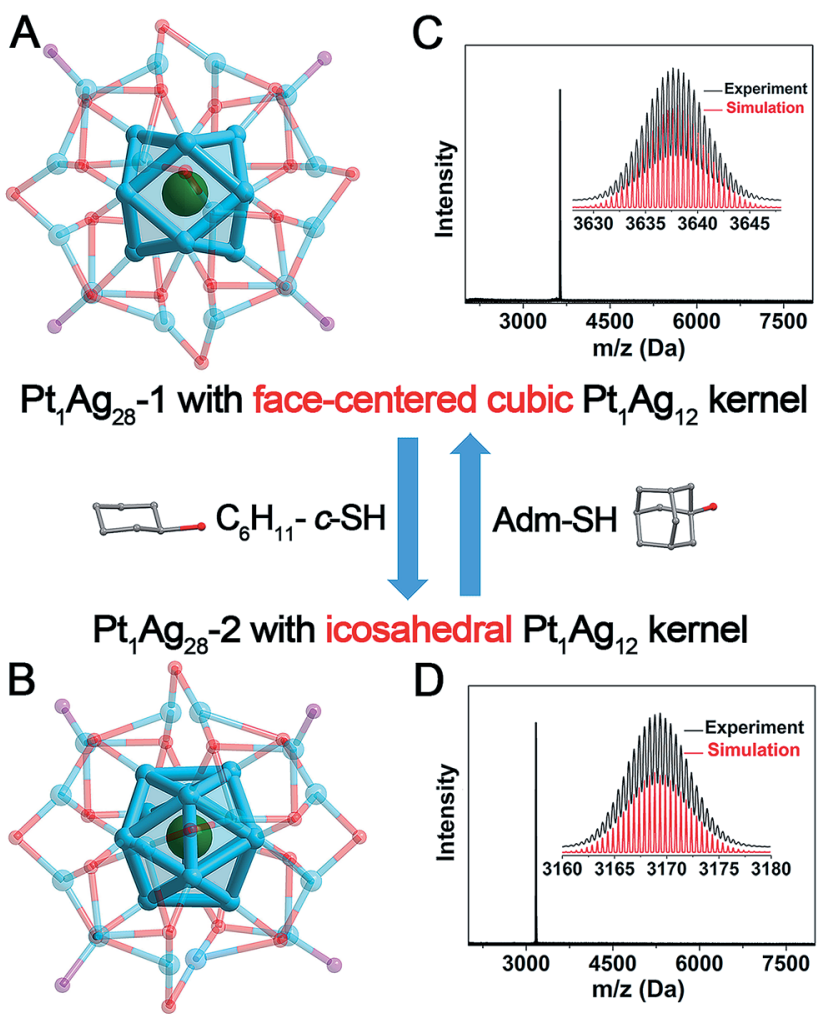

Fig. 2 Illustration of the reversible transformation between $(A)$ the $\mathrm{Pt}_{1} \mathrm{Ag}_{28}-1$ nanocluster with a FCC $\mathrm{Pt}_{1} \mathrm{Ag}_{12}$ kernel and (B) the $\mathrm{Pt}_{1} \mathrm{Ag}_{28}-2$ nanocluster with an icosahedral $\mathrm{Pt}_{1} \mathrm{Ag}_{12}$ kernel induced by the addition of the HS-C- $\mathrm{C}_{6} \mathrm{H}_{11}$ or HS-Adm ligand. ESI-MS spectra of (C) $\mathrm{Pt}_{1} \mathrm{Ag}_{28}-1$ and (D) $\mathrm{Pt}_{1} \mathrm{Ag}_{28}-2$ nanoclusters. Insets: experimental and simulated isotope patterns of each nanocluster. Color codes: green sphere, $\mathrm{Pt}$; blue spheres, Ag; red spheres, S; and purple spheres, P. For clarity, the carbon and hydrogen atoms are not shown. 
Significantly, these two $\mathrm{Pt}_{1} \mathrm{Ag}_{28}$ isomers exhibit reversibility in terms of the kernel configuration between the FCC and icosahedron, which is observed for the first time in the nanocluster range (Fig. 2). Table 1 lists the comparison of bond lengths of Pt(core)-Ag(edge), Ag(edge)-Ag(edge), Ag(edge)-S(motif) and $\mathrm{Ag}$ (vertex)-P(vertex) between these two $\mathrm{Pt}_{1} \mathrm{Ag}_{28}$ isomers (see Fig. S9-S12† for the highlights of these types of bonds). Specifically, in $\mathbf{P t}_{\mathbf{1}} \mathbf{A g}_{\mathbf{2 8}} \mathbf{- 1}$, the bond lengths between the central $\mathrm{Pt}$ atom and the $\mathrm{Ag}$ atoms on the $\mathrm{Pt}_{1} \mathrm{Ag}_{12}$ shell range from 2.768 to $2.797 \AA$ (average: $2.783 \AA$ ), whereas the Pt(core)-Ag(edge) distances in $\mathbf{P t}_{\mathbf{1}} \mathbf{A g}_{\mathbf{2 8}}$-2 shorten to 2.744-2.791 $\AA$ (average: 2.763 $\AA)$. In contrast, the average Ag(edge)-Ag(edge) bond length of $2.801 \AA$ in $\mathbf{P t}_{\mathbf{1}} \mathbf{A g}_{28} \mathbf{- 1}$ significantly increases to $2.915 \AA$ (with a $4.07 \%$ difference) in $\mathbf{P t}_{\mathbf{1}} \mathbf{A g}_{\mathbf{2 8}} \mathbf{- 2}$. Furthermore, the average $\mathrm{Ag}$ (edge)-S bond length displays a $0.92 \%$ elongation in $\mathbf{P} \mathbf{t}_{\mathbf{1}} \mathbf{A g}_{\mathbf{2 8}}$ $\mathbf{2}$ compared with that of the $\mathbf{P t}_{\mathbf{1}} \mathbf{A g}_{\mathbf{2 8}} \mathbf{- 1}$ nanocluster. Moreover, the average $\mathrm{Ag}$ (vertex)-P bond length (2.400 $\AA$ ) in $\mathbf{P t}_{\mathbf{1}} \mathbf{A g}_{\mathbf{2 8}} \mathbf{- 2}$ is also slightly longer than that in $\mathbf{P t}_{\mathbf{1}} \mathbf{A g}_{\mathbf{2 8}} \mathbf{- 1}$ (2.356 ̊).

Electrospray ionization mass spectrometry (ESI-MS) was performed to verify the purity of each $\mathrm{Pt}_{1} \mathrm{Ag}_{28}$ isomer. As shown in Fig. 2C and D, the reaction between $\mathbf{P t}_{\mathbf{1}} \mathbf{A g}_{28} \mathbf{- 1}$ and $\mathrm{HS}-c-\mathrm{C}_{6} \mathrm{H}_{11}$ decreases the mass value from 3637.64 Da to $3169.36 \mathrm{Da}$, which is assigned to $\mathbf{P t}_{\mathbf{1}} \mathbf{A g}_{\mathbf{2 8}}$-2. The magnification of the peak suggests a +2 charge state of $\mathbf{P t}_{\mathbf{1}} \mathbf{A g}_{28}$-2, since this peak evidences a characteristic isotopic pattern with peaks separated by an $\mathrm{m} / \mathrm{z}$ of $0.5 \mathrm{Da}$ (in the positive mode). In this context, the overall charge state of these two $\mathrm{Pt}_{1} \mathrm{Ag}_{28}$ isomers is the same +2 . Of note, the +2 charge state of $\mathbf{P t}_{\mathbf{1}} \mathbf{A g}_{28}$-2 matches the crystal data, because two negative $\left(\mathrm{BPh}_{4}\right)^{-}$counterions were observed in the crystal structure of $\mathbf{P t}_{\mathbf{1}} \mathbf{A g}_{\mathbf{2 8}}$-2. In addition, the gap between the mass peaks of two $\mathrm{Pt}_{1} \mathrm{Ag}_{28}$ isomers is calculated as $936.56 \mathrm{Da}$ (i.e., (3637.64 $3169.36) \times 2$ ), which is in accordance with 18 -fold the molecular weight gap between HS-Adm and HS- $c-\mathrm{C}_{6} \mathrm{H}_{11}$ ligands.

It is accepted that the structures of nanoclusters play a decisive role in their chemical/physical properties. ${ }^{\mathbf{1 a , b , \mathbf { 1 } - \boldsymbol { h }}}$ The optical properties of $\mathrm{Pt}_{1} \mathrm{Ag}_{28}$ isomers are compared here to investigate the precise structure-property correlation. First of all, the time-dependent variation of UV-vis absorption from $\mathbf{P t}_{\mathbf{1}} \mathbf{A g}_{28} \mathbf{- 1}$ to $\mathbf{P t} \mathbf{A}_{\mathbf{1}} \mathbf{A g}_{28}-\mathbf{2}$ was recorded. As shown in Fig. 3A, the absorptions at 545 and $443 \mathbf{n m}$ in $\mathbf{P t}_{\mathbf{1}} \mathbf{A g}_{\mathbf{2 8}} \mathbf{- 1}$ red-shift to 575 and $455 \mathbf{n m}$, respectively, in $\mathbf{P t}_{\mathbf{1}} \mathbf{A g}_{\mathbf{2 8}} \mathbf{- 2}$. Furthermore, the 333 and $305 \mathrm{~nm}$ absorption peaks in $\mathbf{P t}_{\mathbf{1}} \mathbf{A g}_{\mathbf{2 8}} \mathbf{- 1}$ become more pronounced and red-shift to 350 and $315 \mathrm{~nm}$, respectively. A total of four iso-absorption points are observed, centering at

Table 1 Comparison of different bond lengths in $\mathrm{Pt}_{1} \mathrm{Ag}_{28}-1$ and $\mathrm{Pt}_{1} \mathrm{Ag}_{28}-2$ crystal structures

\begin{tabular}{|c|c|c|c|}
\hline Bond length $(\AA)$ & $\mathrm{Pt}_{1} \mathbf{A g}_{28} \mathbf{s}^{-1}$ & $\mathrm{Pt}_{1} \mathbf{A g}_{28^{-2}}$ & Diff. \\
\hline \multirow[t]{2}{*}{ Pt(core)-Ag(edge) } & $2.768-2.797$ & $2.744-2.791$ & \multirow[t]{2}{*}{$0.72 \%$} \\
\hline & Avg. 2.783 & Avg. 2.763 & \\
\hline \multirow[t]{2}{*}{ Ag(edge)-Ag(edge) } & $2.761-2.843$ & $2.819-3.309$ & \multirow[t]{2}{*}{$4.07 \%$} \\
\hline & Avg. 2.801 & Avg. 2.915 & \\
\hline \multirow[t]{2}{*}{ Ag(edge)-S } & $2.438-2.498$ & $2.480-2.517$ & \multirow[t]{2}{*}{$0.92 \%$} \\
\hline & Avg. 2.472 & Avg. 2.495 & \\
\hline \multirow[t]{2}{*}{$\mathrm{Ag}($ vertex $)-\mathrm{P}$} & $2.292-2.384$ & $2.395-2.409$ & \multirow[t]{2}{*}{$1.87 \%$} \\
\hline & Avg. 2.356 & Avg. 2.400 & \\
\hline
\end{tabular}

$307,338,427$ and $454 \mathrm{~nm}$. These iso-absorption points illustrate the high level of conversion from $\mathbf{P t}_{\mathbf{1}} \mathbf{A g}_{\mathbf{2 8}} \mathbf{- 1}$ to $\mathbf{P t}_{\mathbf{1}} \mathbf{A g}_{\mathbf{2 8}} \mathbf{- 2}$. The solution of $\mathbf{P t}_{\mathbf{1}} \mathbf{A g}_{28}$-2 appears green to the naked eye, while the $\mathbf{P t}_{\mathbf{1}} \mathbf{A g}_{28} \mathbf{- 1}$ solution is orange (Fig. 3A, insets). The photon energy plots of the two $\mathrm{Pt}_{1} \mathrm{Ag}_{28}$ isomers were recorded (Fig. 3B), which demonstrate that the energy gap (between the highest occupied molecular orbital (HOMO) and the lowest unoccupied molecular orbital (LUMO)) of $\mathbf{P t}_{\mathbf{1}} \mathbf{A g}_{\mathbf{2 8}} \mathbf{- 2}$ (1.74 eV) is much smaller than that of $\mathbf{P t}_{\mathbf{1}} \mathbf{A g}_{\mathbf{2 8}} \mathbf{- 1}$ (1.86 eV); this is possibly due to the configuration transformation from the FCC to the icosahedron. In addition, accompanied by the ligand-exchange process from $\mathbf{P t}_{\mathbf{1}} \mathbf{A g}_{28} \mathbf{- 1}$ to $\mathbf{P t} \mathbf{t}_{\mathbf{1}} \mathbf{A g}_{28}$-2, gradual reduction was detected in the HOMO-LUMO gap (Fig. 3B).

Fig. $3 \mathrm{C}$ and $\mathrm{D}$ show the $\mathrm{PL}$ performances of the $\mathrm{Pt}_{1} \mathrm{Ag}_{28}$ isomers. First of all, the PL excitation spectra are almost identical to the absorption spectra, which is reminiscent of the behavior of quantum-dots and some nanoclusters. ${ }^{8 b, 13}$ In addition, the emission peak wavelength is not dependent on the excitation wavelength but remains at $672 \mathrm{~nm}$ for $\mathbf{P t}_{\mathbf{1}} \mathbf{A g}_{\mathbf{2 8}} \mathbf{- 1}$ and at $720 \mathrm{~nm}$ for $\mathbf{P t}_{\mathbf{1}} \mathbf{A g}_{\mathbf{2 8}} \mathbf{- 2}$. Furthermore, the emission of each isomer (i.e., $1.84 \mathrm{eV}$ of $\mathbf{P t} \mathbf{A g}_{\mathbf{2}} \mathbf{- 1}$ and $1.72 \mathrm{eV}$ of $\mathbf{P t} \mathbf{A g}_{\mathbf{2 8}} \mathbf{- 2}$ ) is very close to the HOMO-LUMO gap energy derived from the optical absorption spectrum. The extremely small difference in energy illustrates that the fluorescence corresponds to the HOMOLUMO transition of each $\mathrm{Pt}_{1} \mathrm{Ag}_{28}$ isomer.

The structures of nanoclusters are determinant of their physical and chemical properties. ${ }^{\mathbf{1 a}, \boldsymbol{b}, \boldsymbol{f}-\boldsymbol{h}}$ In this context, the significant red-shift of the emission wavelength from $\mathbf{P t}_{\mathbf{1}} \mathbf{A g}_{\mathbf{2 8}} \mathbf{- 1}$ to $\mathbf{P t}_{\mathbf{1}} \mathbf{A g}_{28} \mathbf{- 2}(\sim 50 \mathrm{~nm})$ is traceable to the kernel transformation from the FCC to icosahedron. Considering that the kernel transformation is induced by the ligand exchange, we were motivated to track this configuration variation by matching the ESI-MS changes and the corresponding emission variations. Here, the ESI-MS and fluorescence variations from $\mathbf{P t}_{\mathbf{1}} \mathbf{A g}_{\mathbf{2 8}} \mathbf{- 1}$ to $\mathbf{P t}_{\mathbf{1}} \mathbf{A g}_{\mathbf{2 8}} \mathbf{- 2}$ are detected since the corresponding transformation is much slower than the reverse process, and thus is easier to monitor (see Fig. S13-S15† for the UV-vis, ESI-MS and PL variations from $\mathbf{P t}_{\mathbf{1}} \mathbf{A g}_{28}-\mathbf{2}$ to $\left.\mathbf{P t}_{\mathbf{1}} \mathbf{A g}_{28} \mathbf{- 1}\right)$. First of all, ESI-MS measurements were performed to monitor the ligandexchange degree of each stage from $\mathbf{P t}_{\mathbf{1}} \mathbf{A g}_{\mathbf{2 8}} \mathbf{- 1}$ to $\mathbf{P t}_{\mathbf{1}} \mathbf{A g}_{\mathbf{2 8}} \mathbf{- 2}$ (Fig. 3E), and the time-dependent ligand-exchange process in mild and stepwise modes was deduced. Furthermore, the fluorescence variation can be divided into two periods (Fig. 3F, see the lateral comparison in Fig. S16†): (Period 1) from stage 1 to stage 5: each fluorescence curve only exhibits a single characteristic peak, although the emission red-shifts from $672 \mathrm{~nm}$ of stage 1 to $674,675,677$, and $678 \mathrm{~nm}$ of stages $2-5$, respectively; (Period 2) from stage 6 to stage 9: each emission curve contains two overlapping peaks, where the front peak decreases accompanying the ligand-exchange process, but the rear one increases gradually. Finally, the fluorescence curve just exhibits a single peak at $720 \mathrm{~nm}$, which is the emission of pure $\mathbf{P t}_{\mathbf{1}} \mathbf{A g}_{\mathbf{2 8}} \mathbf{- 2}$ (stage 9). Combining the variations of ESI-MS and fluorescence, the emission just displays a slight red-shift when the number of ligands exchanged on $\mathbf{P t}_{\mathbf{1}} \mathbf{A g}_{\mathbf{2 8}} \mathbf{- 1}$ is less than 9-10 (stages 1-5, depicted by the blue arrow in Fig. $3 \mathrm{~F}$ ). When exchanging more ligands onto the nanocluster, a new peak emerges at $712 \mathrm{~nm}$ 

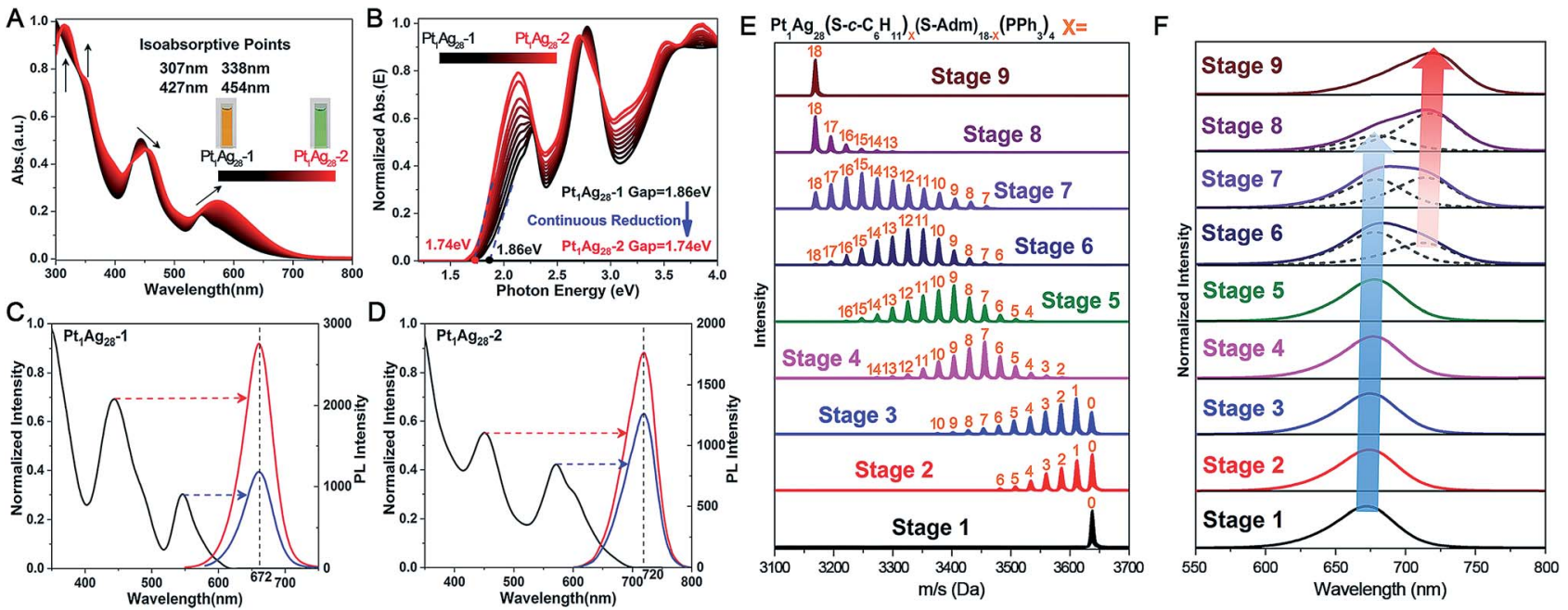

Fig. 3 Real-time alternation of the (A) UV-vis absorption and (B) photon-energy plot from $\mathrm{Pt}_{1} \mathrm{Ag}_{28}-1$ (in black) to $\mathrm{Pt}_{1} \mathrm{Ag}_{28}-2$ (in red). Four isoabsorption points are observed at 307, 338, 427 and $454 \mathrm{~nm}$, as depicted in (A). Insets of (A): photographs of the nanocluster solutions. Excitation spectrum (left) and emission spectra (right) of (C) $\mathrm{Pt}_{1} \mathrm{Ag}_{28}-1$ and (D) $\mathrm{Pt}_{1} \mathrm{Ag}_{28}-2$ at different excitation wavelengths, as indicated by the arrows. Time-dependent (E) ESI-MS spectra and (F) PL emission spectra from $\mathrm{Pt}_{1} \mathrm{Ag}_{28}-1$ to $\mathrm{Pt}_{1} \mathrm{Ag}_{28}-2$. Insets of (F): dotted curves represent the separated overlapped curves. The blue arrow indicates the red-shift and reduction of the emission at $\sim 672 \mathrm{~nm}$. The red arrow indicates the red-shift and enhancement of the emission at $\sim 720 \mathrm{~nm}$.

(stage 6). This new peak gradually red-shifts and increases to the final emission at $720 \mathrm{~nm}$ (stages 6-9, depicted by the red arrow in Fig. $3 \mathrm{~F}$ ), in which process the peak at $\sim 672 \mathrm{~nm}$ fades away. According to these phenomena, we propose that the transformation from $\mathbf{P t}_{\mathbf{1}} \mathbf{A g}_{28} \mathbf{- 1}$ to $\mathbf{P t}_{\mathbf{1}} \mathbf{A g}_{28}$-2 is a two-step process (Fig. 4): (i) the motif transformation process: the outmost motif structures alter gradually when inadequate $\mathrm{S}-c-\mathrm{C}_{6} \mathrm{H}_{11}$ ligands are exchanged on the surface of the $\mathrm{Pt}_{1} \mathrm{Ag}_{28}$ nanocluster. In this process the FCC configuration of the $\mathrm{Pt}_{1} \mathrm{Ag}_{12}$ kernel remains (corresponding to stages 1-5); (ii) the kernel transformation process: sharp transformation of the kernel configuration from the FCC to icosahedron occurs as long as enough foreign ligands have been exchanged on the nanocluster surface, and finally all $\mathrm{Pt}_{1} \mathrm{Ag}_{28}$ nanoclusters contain the icosahedral $\mathrm{Pt}_{1} \mathrm{Ag}_{12}$ kernel (corresponding to stages 6-9). In addition, considering that the exchanged ligands do not come into contact with the innermost $\mathrm{Pt}_{1} \mathrm{Ag}_{12}$ kernel directly, we speculate that the kernel transformation occurs as a result of the motif transformation.

\subsection{XAFS measurements}

Our repeated attempts to crystallize the samples from stages 2-8 were unsuccessful, which might be because such $\mathrm{Pt}_{1} \mathrm{Ag}_{28}$ samples

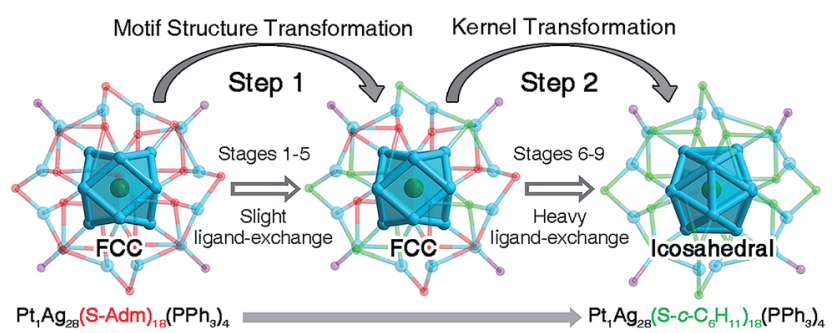

Fig. 4 Proposed two-step transformation from $\mathrm{Pt}_{1} \mathrm{Ag}_{28}-1$ to $\mathrm{Pt}_{1} \mathrm{Ag}_{28}$ - are protected by different proportions of S-Adm and S-c- $\mathrm{C}_{6} \mathrm{H}_{11}$ ligands (Fig. 3E, stages 2-8). XAFS measurements were performed for grasping the structural variation of $\mathrm{Pt}_{1} \mathrm{Ag}_{28}$ nanoclusters in different stages. XAFS results of stages $1,3,5,7$, and 9 are recorded since such results are capable of revealing the structural variations that correspond to the initial PL red-shift $(672 \mathrm{~nm} \rightarrow$ $678 \mathrm{~nm})$ and the further abrupt changes $(678 \mathrm{~nm} \rightarrow 712 \mathrm{~nm})$. As shown in Fig. 5 and Table 2 (see Fig. S17-S21 $\dagger$ for more information), the fitted number of Pt-Ag bonds in each $\mathrm{Pt}_{1} \mathrm{Ag}_{28}$ sample is 12 , which is in accordance with the $\mathrm{Pt}_{1} \mathrm{Ag}_{12}$ kernel in both FCC$\mathrm{Pt}_{1} \mathrm{Ag}_{28}$ and icosahedral- $\mathrm{Pt}_{1} \mathrm{Ag}_{28}$ nanoclusters. Furthermore, the invariable bond lengths of Pt-Ag (remaining as $2.75 \pm 0.01 \AA$, Table 2) in these samples validate the extremely small difference (cal. $0.72 \%$, Table 1) in average $\mathrm{Pt}($ core) $-\mathrm{Ag}(\mathrm{edge})$ bond lengths in $\mathbf{P t}_{\mathbf{1}} \mathbf{A g}_{28^{-1}} \mathbf{1}$ and $\mathbf{P t}_{\mathbf{1}} \mathbf{A g}_{28} \mathbf{2}$.

Importantly, both the $k^{2} \chi(k)$ oscillation and Fourier transform curves of stages 1-5 demonstrate that the Pt-Ag bonds are almost invariable in these stages (Fig. 5 and Table 2); however, the lower intensity of the Fourier transformed EXAFS $k^{2} \chi(k)$ oscillations in stages 1-5 than in stages 7-9 indeed shows the higher disorder degree of Pt-Ag bonds in the former, i.e., the
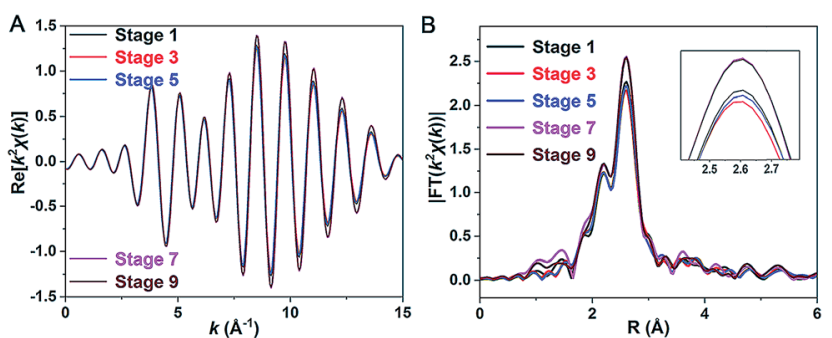

Fig. 5 (A) $k^{2} \chi(k)$ oscillations of $\mathrm{Pt}_{3}$ and (B) corresponding Fourier transforms for $\mathrm{Pt}_{1} \mathrm{Ag}_{28}$ samples at different stages. 
Table 2 Fitted EXAFS results of $\mathrm{Pt}_{1} \mathrm{Ag}_{28}$ samples corresponding to stages 1, 3, 5, 7, and 9 that relate to Fig. 3

\begin{tabular}{llllll}
\hline Stage & Path & $N$ & $R(\AA)$ & $\sigma^{2}\left(10^{-3} \AA^{2}\right)$ & $\Delta E_{0}(\mathrm{eV})$ \\
\hline 1 & Pt-Ag & 12 & $2.75 \pm 0.01$ & $6.9 \pm 0.2$ & $5.5 \pm 0.6$ \\
3 & Pt-Ag & 12 & $2.75 \pm 0.01$ & $7.1 \pm 0.2$ & $5.3 \pm 0.4$ \\
5 & Pt-Ag & 12 & $2.75 \pm 0.01$ & $7.0 \pm 0.1$ & $5.5 \pm 0.4$ \\
7 & Pt-Ag & 12 & $2.75 \pm 0.01$ & $6.3 \pm 0.3$ & $5.7 \pm 0.9$ \\
9 & Pt-Ag & 12 & $2.75 \pm 0.01$ & $6.3 \pm 0.2$ & $5.7 \pm 0.7$
\end{tabular}

larger Debye-Waller factor $\sigma^{2}$ listed in Table 2. Generally, the Debye-Waller factor $\sigma^{2}$ is a sum of two components, thermal disorder $\left({\sigma_{\mathrm{T}}}^{2}\right)$ and structural disorder $\left(\sigma_{\mathrm{S}}^{2}\right)$, i.e., $\sigma^{2}={\sigma_{\mathrm{T}}}^{2}+{\sigma_{\mathrm{S}}}^{2}$. From the single crystal XRD analysis (Table 1), the Pt-Ag bond lengths in $\mathbf{P t}_{\mathbf{1}} \mathbf{A g}_{28} \mathbf{- 1}$ span a narrower range of $0.029 \AA$ (from 2.768 to $2.797 \AA$, average $2.783 \AA)$ than that $(0.047 \AA$, varying from 2.744 to $2.791 \AA$, average $2.763 \AA$ ) in $\mathbf{P t}_{1} \mathbf{A g}_{28}$-2 , indicating a smaller structural disorder $\sigma_{\mathrm{S}}^{2}$ in the FCC isomer. The larger $\sigma^{2}$ but smaller $\sigma_{\mathrm{S}}{ }^{2}$ of the Pt-Ag bonds in $\mathbf{P t}_{\mathbf{1}} \mathbf{A g}_{28^{-1}} \mathbf{1}$ than in icosahedral $\mathbf{P t}_{1} \mathbf{A g}_{28}-\mathbf{2}$ suggests that the thermal disorder $\sigma_{\mathrm{T}}{ }^{2}$ is larger in $\mathbf{P t}_{\mathbf{1}} \mathbf{A g}_{\mathbf{2 8}} \mathbf{8}^{-1}$. It is known that when the measurement temperature $T$ is close to or higher than the Einstein temperature of a bond, the thermal disorder ${\sigma_{\mathrm{T}}}^{2}$ could be approximated by the correlated Einstein model: $\sigma_{\mathrm{T}}^{2}=k_{\mathrm{B}} T / k_{\text {eff }}$, where $k_{\mathrm{B}}$ is the Einstein temperature and $k_{\text {eff }}$ is the effective spring constant of the bond. ${ }^{14}$ Therefore, the larger thermal disorder $\sigma_{\mathrm{T}}{ }^{2}$ in $\mathbf{P t}_{\mathbf{1}} \mathbf{A g}_{28} \mathbf{- 1}$ indicates that the Pt-Ag interaction (reflected by the effective spring constant) is weaker in $\mathbf{P t}_{\mathbf{1}} \mathbf{A g}_{28} \mathbf{- 1}^{\mathbf{1}}$ than in $\mathbf{P t}_{\mathbf{1}} \mathbf{A g}_{28^{-}}$ $\mathbf{2}$, or the Pt-Ag bond is strengthened in $\mathbf{P t}_{\mathbf{1}} \mathbf{A g}_{\mathbf{2 8}} \mathbf{- 2}$. This deduction is supported by the fact that, from the XRD analysis, the average $\mathrm{Pt}-\mathrm{Ag}$ bond length $(2.783 \AA)$ in $\mathbf{P t}_{\mathbf{1}} \mathbf{A g}_{\mathbf{2 8}} \mathbf{- 1}$ is longer by $0.02 \AA$ than that (2.763 $\AA$ ) in $\mathbf{P t}_{\mathbf{1}} \mathbf{A g}_{\mathbf{2 8}} \mathbf{2}^{-2}$. More support could also be afforded by the Kohn-Sham molecular orbitals (MO) of both clusters yielded by DFT calculations (Fig. S22 $\dagger$ ). The bonding HOMO orbitals of $\mathbf{P t}_{1} \mathbf{A g}_{28} \mathbf{- 1}$ are composed of $2.16 \%$ Pt $6 \mathrm{sp}$, $2.29 \%$ Pt 5d, 26.03\% Ag 5sp, 20.3\% Ag 4d, and $41.67 \%$ S 3p orbitals, while the HOMO orbitals of $\mathbf{P t}_{\mathbf{1}} \mathbf{A g}_{\mathbf{2 8}} \mathbf{- 2}$ are composed of 4.08\% Pt 6sp, 1.24\% Pt 5d, 39.79\% Ag 5sp, 21.46\% Ag 4d, and $26.97 \%$ S 3p orbitals. The smaller amount of Pt orbital components in $\mathbf{P t}_{1} \mathbf{A g}_{28}$-1 than in $\mathbf{P t}_{\mathbf{1}} \mathbf{A g}_{28}$-2 (4.45\% versus $\left.5.22 \%\right)$ suggests the weaker Pt-Ag bonding in the former. This is also consistent with the consideration based on antibonding LUMO orbitals, where $\mathbf{P t}_{\mathbf{1}} \mathbf{A g}_{28} \mathbf{- 1}$ has a larger amount of Pt orbital components than $\mathbf{P t}_{\mathbf{1}} \mathbf{A g}_{28}$-2 (8.04\% versus $\left.7.33 \%\right)$.

EXAFS results indeed illustrate that the kernel transformation occurs within the ligand-exchange process from stage 5 to stage 7 . Such a process (stage $5 \rightarrow$ stage 7 ) is also regarded as the break point where significant PL variation occurs (Fig. 3F). In this context, by combining the ESI-MS, PL and EXAFS variation results, it has been unambiguously demonstrated that the nanocluster configuration transformation (from FCC to icosahedron) contains two discrete steps: the motif transformation and the kernel transformation. Specifically, in the early stage of the ligand-exchange process (stages 1-5), only the motif transformation occurs, which hardly affects the inner Pt-Ag bonds; when enough foreign ligands are exchanged on the $\mathrm{Pt}_{1} \mathrm{Ag}_{28}$ nanocluster (stages 5-9), remarkable transformation in the kernel occurs and the FCC configuration turns into the icosahedral configuration.

\subsection{Temperature-dependent PL}

The PL QY of $\mathbf{P t}_{\mathbf{1}} \mathbf{A g}_{28} \mathbf{- 2}$ is only $2.7 \%$ at room temperature, which is much lower than that of $\mathbf{P t}_{\mathbf{1}} \mathbf{A g}_{28} \mathbf{- 1}(\mathrm{QY} \sim 4.9 \%)$. However, $\mathbf{P t}_{\mathbf{1}} \mathbf{A g}_{28^{-2}} \mathbf{2}$ can emit bright-red light at low temperature (QY $100 \%$ at $98 \mathrm{~K}$ or lower temperature). Accordingly, the fluorescence intensity increased significantly (a 63-fold increase by comparing the $98 \mathrm{~K}$ data with the $293 \mathrm{~K}$ data) when the temperature is reduced to $98 \mathrm{~K}$ (Fig. $6 \mathrm{~A}-\mathrm{C}$ ), and the UV-vis absorption presents a 1.8-fold enhancement (Fig. 6D). In this context, the PL QY of $\mathbf{P t}_{1} \mathbf{A g}_{28} \mathbf{z}^{-2}$ increases to almost $100 \%$. In detail, when the temperature is higher than $250 \mathrm{~K}$, the PL intensity is so weak that it cannot be observed by the naked eye (Fig. 6B, inset $275 \mathrm{~K}$ ). When the temperature is continually reduced, the PL intensity enhances rapidly, and an obvious emission can be observed at $173 \mathrm{~K}$ (Fig. 6B, inset $173 \mathrm{~K}$ ). Furthermore, the emission of $\mathbf{P t}_{\mathbf{1}} \mathbf{A g}_{\mathbf{2}} \mathbf{- 2}$ nanoclusters is considerably bright when the temperature is reduced to $98 \mathrm{~K}$ or lower (Fig. 6B, inset $98 \mathrm{~K}$ ). The enhanced PL intensity of $\mathbf{P t}_{1} \mathbf{A g}_{28^{-}}$ 2 is induced by the restrained thermal vibration (non-radiative
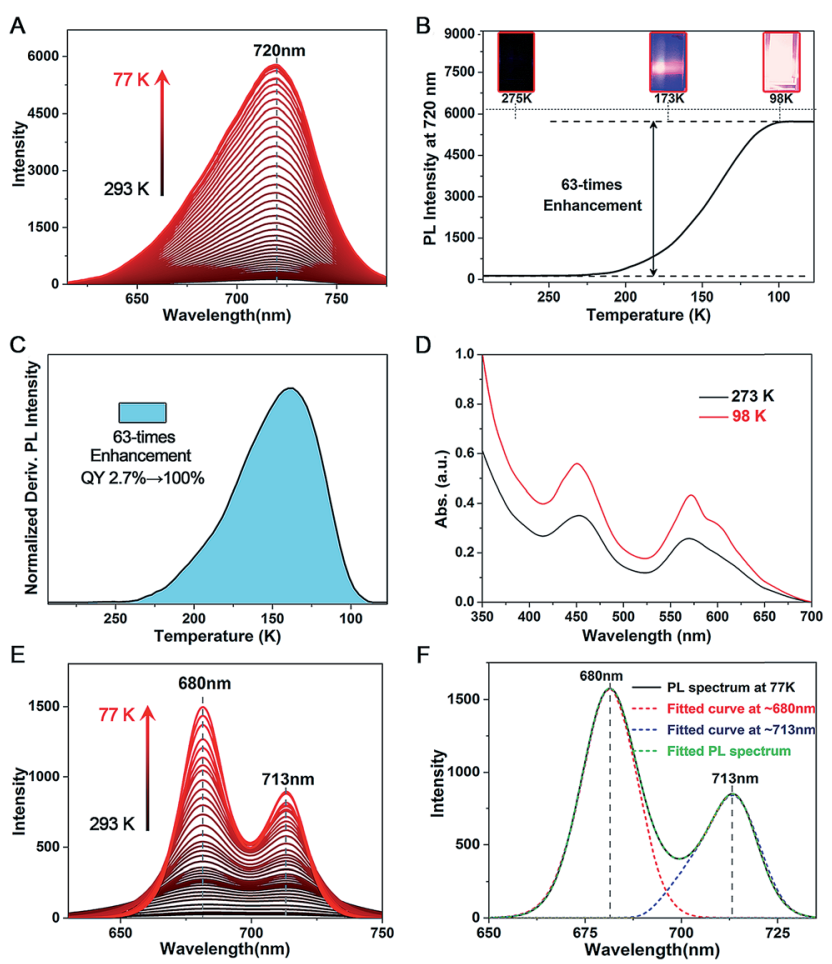

Fig. 6 (A) $\mathrm{PL}$ variation of $\mathrm{Pt}_{1} \mathrm{Ag}_{28}-2$ accompanying the reduction of temperature (from $293 \mathrm{~K}$ to $77 \mathrm{~K}$, monitored per $3 \mathrm{~K}$ ). (B) PL intensity at a fixed wavelength of $720 \mathrm{~nm}$ of $\mathrm{Pt}_{1} \mathrm{Ag}_{28}-2$ at different temperatures. Insets: the digital photographs of $\mathrm{Pt}_{1} \mathrm{Ag}_{28}-2$ solution under $\mathrm{UV}$ light. (C) Derivative result on the $\mathrm{PL}$ intensity of $\mathrm{Pt}_{1} \mathrm{Ag}_{28}-2$. (D) Temperaturedependent UV-vis absorption of $\mathrm{Pt}_{1} \mathrm{Ag}_{28}$-2. (E) $\mathrm{PL}$ variation of the intermediate ligand-exchange product (stage 7) accompanying the reduction of temperature. (F) Fitted curves of the $\mathrm{PL}$ spectrum of the intermediate ligand-exchange product (stage 7) measured at $77 \mathrm{~K}$. 
transition) of the nanoclusters at low temperature, and thus the energy loss is just fluorescence (radiative transition). ${ }^{15}$ For comparison, the PL variation of $\mathbf{P t}_{\mathbf{1}} \mathbf{A g}_{28} \mathbf{- 1}$ accompanying the reduction of the temperature is exhibited in Fig. S23. $\dagger$

Temperature-dependent PL spectra were also measured on the intermediate ligand-exchange $\mathrm{Pt}_{1} \mathrm{Ag}_{28}$ product. Specifically, we chose the product of stage 7 (see Fig. 3E and F) because the PL spectrum of this product displays the most obvious overlapping peaks. As shown in Fig. 6E, accompanying the reduction of temperature from $293 \mathrm{~K}$ to $77 \mathrm{~K}$, the overlapped peaks become sharper and more separated. Meanwhile, the emission intensity appears to be significantly enhanced in the temperature reduction process. In this context, the peak-fitting becomes more easy and precise. Fig. $6 \mathrm{~F}$ exhibits the overlapped curves from the PL spectrum of the intermediate ligand-exchange product (stage 7) measured at $77 \mathrm{~K}$. Two independent curves were separated from the multi-peak PL spectrum, and they center at 680 and $713 \mathrm{~nm}$, respectively. Of note, the fitting-peak positions are retained for these two separated curves; however, following the temperature reduction, the relative intensity of the fitted curve at $\sim 680 \mathrm{~nm}$ becomes stronger relative to the other one, which demonstrates the different emission performance of the FCC- $\mathrm{Pt}_{1} \mathrm{Ag}_{28}$ and icosahedral-Pt ${ }_{1} \mathrm{Ag}_{28}$ nanoclusters with the temperature reduction.

\section{Conclusions}

In summary, a ligand-exchange method was exploited to reversibly transform the $\mathbf{P t}_{\mathbf{1}} \mathbf{A g}_{\mathbf{2 8}} \mathbf{- 1}$ nanoclusters with a FCC configuration and the $\mathbf{P t}_{\mathbf{1}} \mathbf{A g}_{\mathbf{2}} \mathbf{2}$-2 nanoclusters with an icosahedral configuration. This is the first time that the isomerism phenomenon with reversible configurations has been observed. ESI-MS, PL and EXAFS results were combined to illustrate that the configuration transformation (between FCC and icosahedron) is a two-step process, including the outmost motif transformation process and the innermost kernel transformation process, where the latter transformation is induced by the former one. Based on these $\mathrm{Pt}_{1} \mathrm{Ag}_{28}$ isomers, the corresponding structure-optical property correlation was evaluated. UV-vis absorption, together with PL emission, demonstrates a reduced HOMO-LUMO gap of $\mathbf{P t}_{\mathbf{1}} \mathbf{A g}_{\mathbf{2 8}} \mathbf{- 2}$ compared with that of $\mathbf{P t}_{\mathbf{1}} \mathbf{A g}_{\mathbf{2 8}} \mathbf{- 1}$. Overall, this work presents a nanocluster isomer system with a reversibly transforming configuration, which hopefully draws great attention of structural and theoretical chemists to fully understand the structural transformations as well as the structure-property correlation of metal nanoclusters.

\section{Conflicts of interest}

There are no conflicts to declare.

\section{Acknowledgements}

We acknowledge the financial support by the NSFC (U1532141, 21631001, 21871001, 21803001, U1632263), the Ministry of Education, the Education Department of Anhui Province (KJ2017A010), and the 211 Project of Anhui University.

\section{Notes and references}

1 (a) R. Jin, C. Zeng, M. Zhou and Y. Chen, Chem. Rev., 2016, 116, 10346; (b) I. Chakraborty and T. Pradeep, Chem. Rev., 2017, 117, 8208; (c) Q. Yao, X. Yuan, T. Chen, D. T. Leong and J. Xie, Adv. Mater., 2018, 30, 1802751; (d) B. Bhattarai, Y. Zaker, A. Atnagulov, B. Yoon, U. Landman and T. P. Bigioni, Acc. Chem. Res., 2018, 51, 3104; (e) P. Liu, R. Qin, G. Fu and N. Zheng, J. Am. Chem. Soc., 2017, 139, 2122; (f) M. Agrachev, M. Ruzzi, A. Venzo and F. Maran, Acc. Chem. Res., 2019, 52, 44; (g) K. Kwak and D. Lee, Acc. Chem. Res., 2019, 52, 12; (h) B. Nieto-Ortega and T. Bürgi, Acc. Chem. Res., 2018, 51, 2811; (i) K. L. D. M. Weerawardene, H. Häkkinen and C. M. Aikens, Annu. Rev. Phys. Chem., 2018, 69, 205; (j) W. W. Xu, X. C. Zeng and Y. Gao, Acc. Chem. Res., 2018, 51, 2739; ( $k$ ) Q. Tang, G. Hu, V. Fung and D.-e. Jiang, Acc. Chem. Res., 2018, 51, 2793; (l) N. A. Sakthivel and A. Dass, Acc. Chem. Res., 2018, 51, 1774; ( $m$ ) Z. Lei, X.-K. Wan, S.-F. Yuan, Z.-J. Guan and Q.-M. Wang, Acc. Chem. Res., 2018, 51, 2465; (n) J. Yan, B. K. Teo and N. Zheng, Acc. Chem. Res., 2018, 51, 3084; (o) T. Zhao, P. J. Herbert, H. Zheng and K. L. Knappenberger, Acc. Chem. Res., 2018, 51, 1433; (p) X. Kang and M. Zhu, Chem. Soc. Rev., 2019, 48, 2422.

2 (a) P. D. Jadzinsky, G. Calero, C. J. Ackerson, D. A. Bushnell and R. D. Kornberg, Science, 2007, 318, 430; (b) A. Desireddy, B. E. Conn, J. Guo, B. Yoon, R. N. Barnett, B. M. Monahan, K. Kirschbaum, W. P. Griffith, R. L. Whetten, U. Landman and T. P. Bigioni, Nature, 2013, 501, 399; (c) S. Takano, H. Hirai, S. Muramatsu and T. Tsukuda, J. Am. Chem. Soc., 2018, 140, 12314; (d) T.-A. D. Nguyen, Z. R. Jones, B. R. Goldsmith, W. R. Buratto, G. Wu, S. L. Scott and T. W. Hayton, J. Am. Chem. Soc., 2015, 137, 13319; (e) S. Sharma, K. K. Chakrahari, J.-Y. Saillard and C. W. Liu, Acc. Chem. Res., 2018, 51, 2475; (f) S. Hossain, Y. Niihori, L. V. Nair, B. Kumar, W. Kurashige and Y. Negishi, Acc. Chem. Res., 2018, 51, 3114; $(g)$ R.-W. Huang, Y.-S. Wei, X.-Y. Dong, X.-H. Wu, C.-X. Du, S.-Q. Zang and T. C. W. Mak, Nat. Chem., 2017, 9, 689; (h) B. Du, X. Jiang, A. Das, Q. Zhou, M. Yu, R. Jin and J. Zheng, Nat. Nanotechnol., 2017, 12, 1096; (i) M. Sugiuchi, Y. Shichibu and K. Konishi, Angew. Chem., Int. Ed., 2018, 57, 7855; (j) C. A. Hosier and C. J. Ackerson, J. Am. Chem. Soc., 2019, 141, 309; (k) Y. Lin, P. Charchar, A. J. Christofferson, M. R. Thomas, N. Todorova, M. M. Mazo, Q. Chen, J. Doutch, R. Richardson, I. Yarovsky and M. M. Stevens, J. Am. Chem. Soc., 2018, 140, 18217; (l) G. Panapitiya, G. Avendaño-Franco, P. Ren, X. Wen, Y. Li and J. P. Lewis, J. Am. Chem. Soc., 2018, 140, 17508.

3 (a) H.-E. Lee, H.-Y. Ahn, J. Mun, Y. Y. Lee, M. Kim, N. H. Cho, K. Chang, W. S. Kim, J. Rho and K. T. Nam, Nature, 2018, 556, 360; (b) W. Ma, L. Xu, A. F. de Moura, X. Wu, H. Kuang, C. Xu and N. A. Kotov, Chem. Rev., 2017, 117, 8041; (c) H. Kanai, S. B. Choe and K. J. Klabunde, J. Am. Chem. Soc., 1986, 108, 2019; (d) S. B. Choe, H. Kanai and K. J. Klabunde, J. Am. Chem. Soc., 1989, 111, 2875; (e) K. J. Klabunde, 
T. Groshens, H. F. Efner and M. J. Kramer, J. Organomet. Chem., 1978, 157, 91.

4 (a) J. Yan, H. Su, H. Yang, C. Hu, S. Malola, S. Lin, B. K. Teo, H. Häkkinen and N. Zheng, J. Am. Chem. Soc., 2016, 138, 12751; (b) Y. Zhu, H. Wang, K. Wan, J. Guo, C. He, Y. Yu, L. Zhao, Y. Zhang, J. Lv, L. Shi, R. Jin, X. Zhang, X. Shi and Z. Tang, Angew. Chem., Int. Ed., 2018, 57, 9059; (c) G. Deng, S. Malola, J. Yan, Y. Han, P. Yuan, C. Zhao, X. Yuan, X. S. Lin, Z. Tang, B. K. Teo, H. Häkkinen and N. Zheng, Angew. Chem., Int. Ed., 2018, 57, 3421; (d) H. Yang, J. Yan, Y. Wang, G. Deng, H. Su, X. Zhao, C. Xu, B. K. Teo and N. Zheng, J. Am. Chem. Soc., 2017, 139, 16113; (e) S. Li, X.-S. Du, B. Li, J.-Y. Wang, G.-P. Li, G.-G. Gao and S.-Q. Zang, J. Am. Chem. Soc., 2018, 140, 594.

5 (a) I. Dolamic, B. Varnholt and T. Bürgi, Nat. Commun., 2015, 6, 7117; (b) I. Dolamic, S. Knoppe, A. Dass and T. Bürgi, Nat. Commun., 2012, 3, 798; (c) S. Takano and T. Tsukuda, J. Phys. Chem. Lett., 2016, 7, 4509; (d) N. V. Karimova and C. M. Aikens, J. Phys. Chem. C, 2018, 122, 11051; (e) M. Zhu, H. Qian, X. Meng, S. Jin, Z. Wu and R. Jin, Nano Lett., 2011, 11, 3963; (f) Q. Tang, R. Ouyang, Z. Tian and D.-e. Jiang, Nanoscale, 2015, 7, 2225.

6 (a) S. Tian, Y.-Z. Li, M.-B. Li, J. Yuan, J. Yang, Z. Wu and R. Jin, Nat. Commun., 2015, 6, 8667; (b) J. Chen, L. Liu, X. Liu, L. Liao, S. Zhuang, S. Zhou, J. Yang and Z. Wu, Chem.-Eur. J., 2017, 23, 18187; (c) S. Zhuang, L. Liao, M.-B. Li, C. Yao, Y. Zhao, H. Dong, J. Li, H. Deng, L. Lie and Z. Wu, Nanoscale, 2017, 9, 14809; (d) C. Zeng, T. Li, A. Das, N. L. Rosi and R. Jin, J. Am. Chem. Soc., 2013, 135, 10011; (e) Y. Chen, C. Liu, Q. Tang, C. Zeng, T. Higaki, A. Das, D.-e. Jiang, N. L. Rosi and R. Jin, J. Am. Chem. Soc., 2016, 138, 1482; (f) S. Jin, F. Xu, W. Du, X. Kang, S. Chen, J. Zhang, X. Li, D. Hu, S. Wang and M. Zhu, Inorg. Chem., 2018, 57, 5114; $(g)$ S. Zhuang, L. Liao, J. Yuan, N. Xia, Y. Zhao, C. Wang, Z. Gan, N. Yan, L. He, J. Li, H. Deng, Z. Guan, J. Yang and Z. Wu, Angew. Chem., Int. Ed., 2019, 58,4510 .

7 (a) N. A. Sakthivel, M. Stener, L. Sementa, A. Fortunelli, G. Ramakrishna and A. Dass, J. Phys. Chem. Lett., 2018, 9, 1295; (b) T. Higaki, M. Zhou, K. J. Lambright, K. Kirschbaum, M. Y. Sfeir and R. Jin, J. Am. Chem. Soc., 2018, 140, 5691; (c) Q. Yao, X. Yuan, V. Fung, Y. Yu, D. T. Leong, D.-e. Jiang and J. Xie, Nat. Commun., 2017, 8, 927.
8 (a) L. Liao, S. Zhuang, C. Yao, N. Yan, J. Chen, C. Wang, N. Xia, X. Liu, M.-B. Li, L. Li, X. Bao and Z. Wu, J. Am. Chem. Soc., 2016, 138, 10425; (b) X. Kang, M. Zhou, S. Wang, S. Jin, G. Sun, M. Zhu and R. Jin, Chem. Sci., 2017, 8, 2581; (c) H. Dong, L. Liao and Z. Wu, J. Phys. Chem. Lett., 2017, 8, 5338; (d) C. Zeng, H. Qian, T. Li, G. Li, N. L. Rosi, B. Yoon, R. N. Barnett, R. L. Whetten, U. Landman and R. Jin, Angew. Chem., Int. Ed., 2012, 51, 13114.

9 J. P. Perdew, K. Burke and M. Ernzerhof, Phys. Rev. Lett., 1996, 77, 3865.

10 E. J. Baerends, T. Ziegler, J. Autschbach, D. Bashford, A. Bérces, F. M. Bickelhaupt, C. Bo, P. M. Boerrigter, L. Cavallo, D. P. Chong, L. Deng, R. M. Dickson, D. E. Ellis, M. van Faassen, L. Fan, T. H. Fischer, C. Fonseca Guerra, M. Franchini, A. Ghysels, A. Giammona, S. J. A. van Gisbergen, A. W. Götz, J. A. Groeneveld, O. V. Gritsenko, M. Grüning, S. Gusarov, F. E. Harris, P. van den Hoek, C. R. Jacob, H. Jacobsen, L. Jensen, J. W. Kaminski, G. van Kessel, F. Kootstra, A. Kovalenko, M. V. Krykunov, E. van Lenthe, D. A. McCormack, A. Michalak, M. Mitoraj, S. M. Morton, J. Neugebauer, V. P. Nicu, L. Noodleman, V. P. Osinga, S. Patchkovskii, M. Pavanello, P. H. T. Philipsen, D. Post, C. C. Pye, W. Ravenek, J. I. Rodríguez, P. Ros, P. R. T. Schipper, G. Schreckenbach, J. S. Seldenthuis, M. Seth, J. G. Snijders, M. Solà, M. Swart, D. Swerhone, G. te Velde, P. Vernooijs, L. Versluis, L. Visscher, O. Visser, F. Wang, T. A. Wesolowski, E. M. van Wezenbeek, G. Wiesenekker, S. K. Wolff, T. K. Woo and A. L. Yakovlev. ADF2013, SCM, Theoretical Chemistry, Vrije Universiteit, Amsterdam, The Netherlands, http://www.scm.com.

11 S. Chen, L. Xiong, S. Wang, Z. Ma, S. Jin, H. Sheng, Y. Pei and M. Zhu, J. Am. Chem. Soc., 2016, 138, 10754.

12 S. Yang, J. Chai, Y. Song, J. Fan, T. Chen, S. Wang, H. Yu, X. Li and M. Zhu, J. Am. Chem. Soc., 2017, 139, 5668.

13 (a) H. Wu, H. Zhu, J. Zhuang, S. Yang, C. Liu and Y. C. Cao, Angew. Chem., Int. Ed., 2008, 47, 3730; (b) S. Wang, X. Meng, A. Das, T. Li, Y. Song, T. Cao, X. Zhu, M. Zhu and R. Jin, Angew. Chem., Int. Ed., 2014, 53, 2376.

14 A. I. Frenkel and J. J. Rehr, Phys. Rev. B: Condens. Matter Mater. Phys., 1993, 48, 585.

15 (a) X. Kang, S. Wang and M. Zhu, Chem. Sci., 2018, 9, 3062; (b) X. Wei, X. Kang, Q. Yuan, C. Qin, S. Jin, S. Wang and M. Zhu, Chem. Mater., 2019, 31, 4945. 